\title{
ANALYSIS OF CANDIDATE SNPS IN PATIENTS WITH GENETIC GENERALIZED EPILEPSY
}

\author{
Felipe S. Kaibara*, Iscia Lopes-Cendes, Rodrigo Secolin.
}

\begin{abstract}
Genome-wide association studies (GWAS) and exome sequencing have identified single nucleotide polymorphisms (SNPs) associated with genetic generalized epilepsy (GGE). However, these studies were based on European and Chinese populations, and the SNPs found could not be useful for polygenetic risk assessment in underrepresented populations as admixed Americans. Therefore, the purpose of this study is to evaluate whether these candidate SNPs for susceptibility to GGE are also found to be associated with the phenotype in a Brazilian GGE cohort.
\end{abstract}

\section{Keywords:}

Neurology, Genetic Generalized Epilepsy, Association Study

\section{Introduction}

Genetic Generalized epilepsy (GGE) is the most common type of generalized epilepsy, whose main characteristic is the occurrence of recurrent seizures with no specific region of origin in the brain. In addition, the seizure spreads rapidly along both brain hemispheres (1). Currently, the diagnosis of GGE relies on clinical information and electroencephalographic examination.

Evidence of genetic factors has been extensively reported in patients with GGE. In this context, GWASs have identified 52 genetic variants that predispose to GGE, which are generally located in genes encoding ionic channels and synaptic vesicles.

However, these reported studies were based mostly on European and Chinese populations, and the GWAS results could not be transferable to admixed American populations (2). Therefore, the present study aims to assess the existence of an association between the 52 candidate genetic variants and the disease phenotype in Brazilian patients with GGE.

\section{Results and Discussion}

We used data from SNP-array genotypes of 427 individuals, including 87 patients with GGE and 340 controls. Among the 52 candidate variants identified for GGE in the literature, we found 17 in the SNP-array dataset. In order to assess the statistical power to detect genetic association between the SNPs and GGE, we estimated the statistical power and effect size by the G*POWER v.3.1.9.2 software, according to the following parameters: logistic regression; two-tail; probability of alpha error $p=0.003$ (adjusted for 17 multiple tests); statistical power $=0.8$; sample total size $=427$; other parameters default. The results of the statistical power show that the sample has $80 \%$ of chance of detecting a genetic association with effect size, estimated by odds ratio, lower than 0.64 (for protection against the disease) or higher than 1.56. (for increased susceptibility to the disease)

All 17 candidate SNPs presented allelic frequencies higher than 0.01 in Brazilian control individuals. However, four SNPs were not in Hardy-Weinberg equilibrium $(p<0.01 ; \quad r s 12059546, \quad r s 12185644, \quad r s 1332470$, and rs 1046276). Therefore, these were not considered suitable for further analysis, since they might present spurious results because of biased genotypic distribution due to evolutional processes rather than the association itself.
According to the logistic regression analysis, one SNP showed evidence of association with GGE (rs9788, nominal $\mathrm{p}=0.03663$ (OR=1.44; IC95\%=1.02 - 2.08). However, the result did not hold after Bonferroni's correction for multiple tests (corrected $p=0.6226$ ). Interestingly, the SNP rs1046276 also showed evidence of association, even after the correction for multiple tests (nominal $\mathrm{p}=0.00039$; corrected $\mathrm{p}=0.00536 ; \mathrm{OR}=0.42$; IC95\% $=0.36-0.75)$. However, this last SNP is not in Hardy-Weinberg equilibrium, and it is not possible to determine if the difference in the genotypic distribution is due to a positive association with or it is just a result of the evolutionary processes and population demography.

\section{Conclusions}

The assessment of the 17 candidate SNPs available in the SNP-array did not show evidence for association in Brazilian patients with GGE. Since these SNPs were previously related to GGE in other populations, we suggest that the genetic structure of the Brazilian patients with GGE could be different in comparison with European and Chinese patients. Our results strengthen the hypothesis of non-transferability of GWAS results from one population to another. However, even though we presented enough statistical power to detect association using the Brazilian cohort with GGE, a larger sample size would be necessary to access better the results obtained for SNPs rs9788 and rs 1046276.

It was also important to point it out that there are additional 35 candidate SNPs which were not available in the SNP-array dataset. Therefore, the next step of this project will be the imputation of these 35 SNPs from the SNP-array dataset, which is a computational and statistical process that identifies SNPs stemming from a different source of genome data (such as the 1000 Genome Project), according to parameters such as linkage disequilibrium.

\section{Acknowledgement}

This project is supported by CEPID-BRAINN, FAPESP.

1. Berg AT, Berkovic SF, Brodie MJ, Buchhalter J, Cross JH, Van Emde Boas W, et al. Revised terminology and concepts for organization of seizures and epilepsies: Report of the ILAE Commission on Classification and Terminology, 2005-2009. Epilepsia. 2010;51(4):676-85.

2. Martin AR, Gignoux CR, Walters RK, Wojcik GL, Neale BM, Gravel S, et al. Human Demographic History Impacts Genetic Risk Prediction across Diverse Populations. The American Journal of Human Genetics. 2017;100(4):1-15. 